\title{
Deux inscriptions latines inédites
}

\author{
par ANNA SADURSKa
}

Parmi la dizaine d'inseriptions latines inédites en Pologne j'ai choisi deux épitaphes accompagnées par les portraits des défunts. Je crois que cette catégorie de monuments est particulièrement destinée à une étude à la fois épigraphique et archéologique, c'est à dire la méthode postulée par le Professeur Günther Klaffenbach, ${ }^{1}$ auquel je suis heureuse de pouvoir offrir ces quelques pages.

1. Epitaphe de deux soeurs, fig. 1.

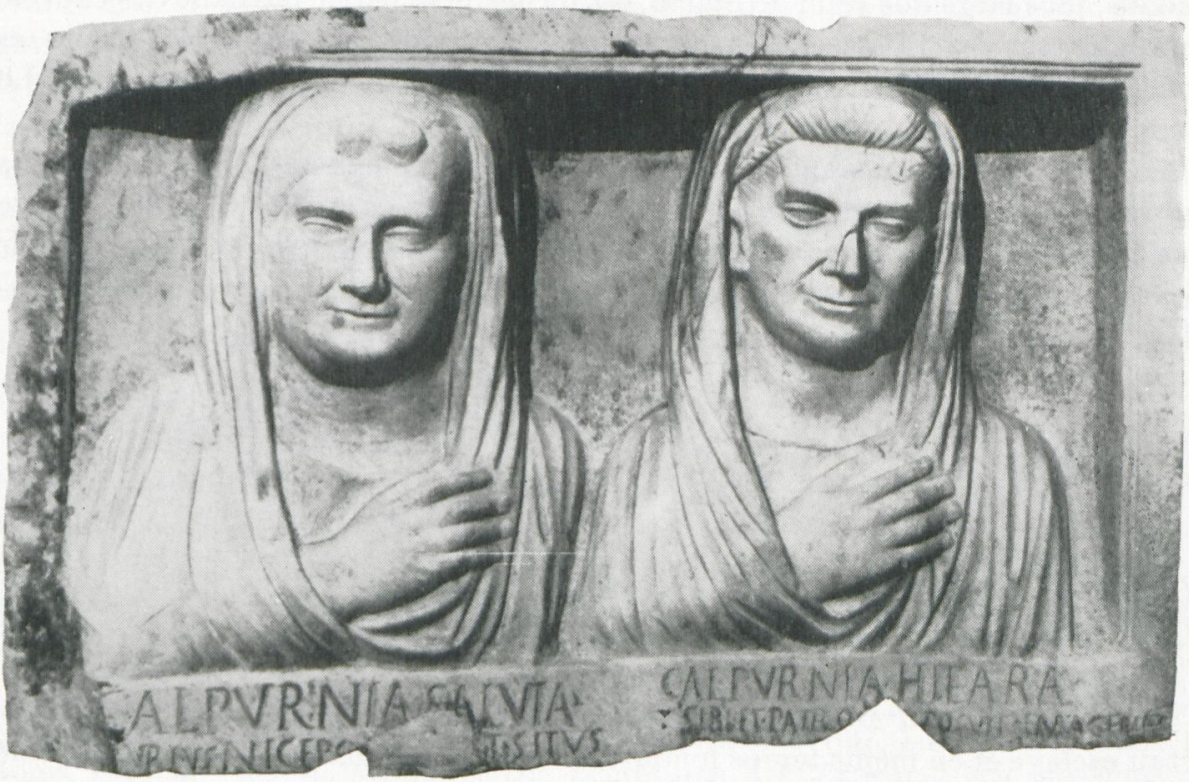

Fig. 1

La pièce se trouve au Musée National de Cracovie annexe Czartoryski.² Grâce aux recherches d'une jeune chercheuse polonaise, nous sommes bien

1 G. Klaffenbach, Archäologie und Epigraphik, AA 1948/49, col. 253-255.

${ }^{2}$ Inv. $\mathrm{n}^{\circ}$ DMNK Cz. 2034. Marbre blane à grain fin avee des veines bleues. H. 0,61 m., larg. 0,90 m., ép. 0,17 m., h. des lettres 0,01-0,03 m. Manquent les nez (restaurés en marbre) et quelques lettres de l'inscription. 
informés sur la provenance. La stèle a été achetée à Rome en 1830 chez Ignazio Vescovali par le comte Artur Potocki pour son château de Krzeszowice. ${ }^{1}$ Ainsi la provenance romaine semble certaine.

L'inscription se trouve sur une stèle rectangulaire, avec deux bustes féminins en relief dans une niche rectangulaire. Les deux femmes, dont celle de gauche est probablement plus jeune et plus forte, sont vêtues de palla qui leur couvrent la tête et enveloppent le corps en ne laissant libre que la main droite. Sous ce vêtement est visible la stola qui forme un décolleté rond. Les deux dames posent la main droite sur le coeur. Toutes les deux ont un sourire tranquille. Les visages et les coiffures sont différenciés. Le visage de la dame de gauche, Calpurnia Salvia, est très large, avec un double menton et un front large mais bas. Sa coiffure, très à la mode à Rome vers le début de l'Empire, est simple: les cheveux, divisés en deux sur le devant, forment deux nattes minces et un petit nodus double qui couvre la racine des nattes. La masse des cheveux formait sans doute un chignon sur la nuque. La coiffure de la femme de droite, Calpurnia Hilara, est plus élégante. Son front haut est partiellement couvert par une frange frisée. Elle est coiffée en une couronne formée de deux nattes croisées. Le nodus, plus large que celui de Salvia, est formé de la partie médiane des cheveux enroulés sur les tresses de la couronne. Le visage de Calpurnia Hilara est maigre, les joues creuses, les pommettes saillantes et le menton pointu. Les autres traits de ces deux visages sont ressemblants.

L'inscription, au-dessous des bustes, est en deux lignes. Le premier vers se rapporte aux deux portraits, le second à deux hommes qui étaient enterrés avec les Calpurniae. Néanmoins il est bien visible sur la pierre que nous avons à faire ici à deux inscriptions gravées l'une à gauche, l'autre à droite et c'est ainsi qu'il faut les lire.

Texte:

CALPVRNIA. SALVIA.

.. VRIVSNICEPO .... T.SITVS
CALPVRNIA. HILARA. SIBI.ET.PATRO . . POSVIT. EMAGENES

Transeription:

Calpurnia Salvia. / [Sp]urius Nicepo[r hic es]t situs.

Calpurnia Hilara. / Sibi et patro[nis] posuit Emagenes.

Nicepor: CIL VI 9456. 6354.

Calpurnia Salvia et Calpurnia Hilara étaient sans doute sœurs. Emagenes était esclave et en même temps fondateur de la stèle. Puisque celle-là est destinée aux deux dames j'ai mis le pluriel dans la seconde ligne de la seconde inscription. L'esclave appartenait probablement aux deux sœurs et celle qui vécut plus longtemps, Hilara sans doute, l'a chargé de cette tâche. Il a reçu en revanche le droit d'être enterré dans le tombeau de famille. Le quatrième personnage enterré dans ce tombeau, mentionné dans l'inscription, reste énigmatique.

1 O. Hirsch, O pochodzeniu kolekeji antyków z Krzeszowic, Meander 20, 1965, 311-312 n. 14. Le document cité ibidem: Ms. Pot. D. 131, Archive des Actes Anciens de Cracovie. 
Spurius Nicepor était un enfant naturel de condition libre. Son épitaphe semble être plus étroitement liée avec celle de Salvia, mais cela put aussi être question de la place laissée libre pour une inscription. On serait tout de même tenté de risquer l'hypothèse qu'il s'agit d'un fils de Calpurnia Salvia. Les deux hommes portent des surnoms grecs. Les surnoms féminins sont latins, mais vu les relations étroites entre les deux dames et les deux Grecs, dont l'un est esclave et l'autre enfant naturel, il est à croire que toute la famille appartenait au milieu des affranchis grees de Rome. Ajoutons que toutes les Calpurniae Salviae et Calpurniae Hilarae de Rome étaient des affranchies, cf. CIL VI 9872. 14164. 33837. 14145. 14232a. 21892.9143 .9576 a.

Cette supposition est confirmée par la forme de la stèle. Elle appartient à une série de monuments funéraires romains qui sont datés entre le second quart du $I^{e r}$ siècle avant $n$. è. et le début du règne de Tibère, destinés pour la plupart à des affranchis. Le vérisme très rigoureux, le modelé linéaire, le style qui tient encore de la sculpture républicaine, caractérisent ce groupe. Citons les stèles les plus connues: celle des Furii au Museo Profano Lateranense, datée à 40-30; des Servilii, ibidem, de l'époque d'Auguste; une stèle d'Ostie, datée vers 25 avant n. è. et celle des Vibii, au. Museo Vaticano, probablement du temps d'Auguste. ${ }^{1}$

La stèle des Calpurniae à Cracovie fut exécutée sans doute en cette période. La coiffure des deux dames imite celle de Julia Augusti, attestée par les monnaies entre 20 et 2 avant $n$. è., et après l'exil de Julie par les portraits privés en ronde bosse. Elle resta en usage jusqu'à vers 20 de n. è., mais seulement parmi les femmes âgées et peu élégantes. ${ }^{2}$ Puisque les deux Calpurniae sont évidemment à ranger dans cette catégorie, la date de la stèle ne peut être mieux précisée qu'entre 10 avant $n$. è. et 20 de n. è., probablement vers la fin de cette période.

2. Epitaphe de la femme d'un esclave, figs. 2. 3.

L'épitaphe est gravée sur un autel funéraire qui se trouve au château de Nieborów annexe du Musée National de Varsovie. ${ }^{3}$ L'histoire de la collection des antiques de Nieborów a été élucidée par K. Michalowski, mais le monument en question n'est mentionné dans aucun document. Il nous reste à supposer que l'autel a été acheté ou bien reçu par Helena Radziwiłt, avec d'autres marbres antiques, entre 1778 et $1821 .{ }^{4} \mathrm{Il}$ provient de Rome.

1 A. Giuliano, Catalogo dei Ritratti Romani del Museo Profano Lateranense, Città del Vaticano 1957, resp. $\mathrm{n}^{\circ}$ 2. $\mathrm{n}^{\circ}$ 3. R. Calza, Scavi di Ostia 5; I Ritratti 1, Roma 1964, $\mathrm{n}^{\circ}$ 36; W. Amelung, Die Sculpturen des Vaticanischen Museums 1, Berlin 1903, pl. 36 (Chiaramonti $60 \mathrm{E}$ ).

${ }^{2}$ L. Furnée - van Zwet, Fashion in Women's Hair-dress in the First Century of the Roman Empire, B A Besch 31, 1956, 7.

${ }^{3}$ Nieborów, actuellement dans les réserves, sans $\mathbf{n}^{0}$ inv. Marbre blanc à grain fin. H. $0,575 \mathrm{~m}$., larg. $0,39 \mathrm{~m}$., ép. 0,32 ., h. des lettres $0,02 \mathrm{~m}$. La surface très tachée et partiellement décomposée. L'autel cassé et recollé, le collage coupe la face frontale. Les nez dans les portraits endommagés.

4 K. Michałowski, Zbiór antyków grecko-rzymskich w Nieborowie jako wyraz kolekcjonerstwa polskiego w dobie Oświecenia, Biuletyn Historii Sztuki 13, 1951, 124-137. 


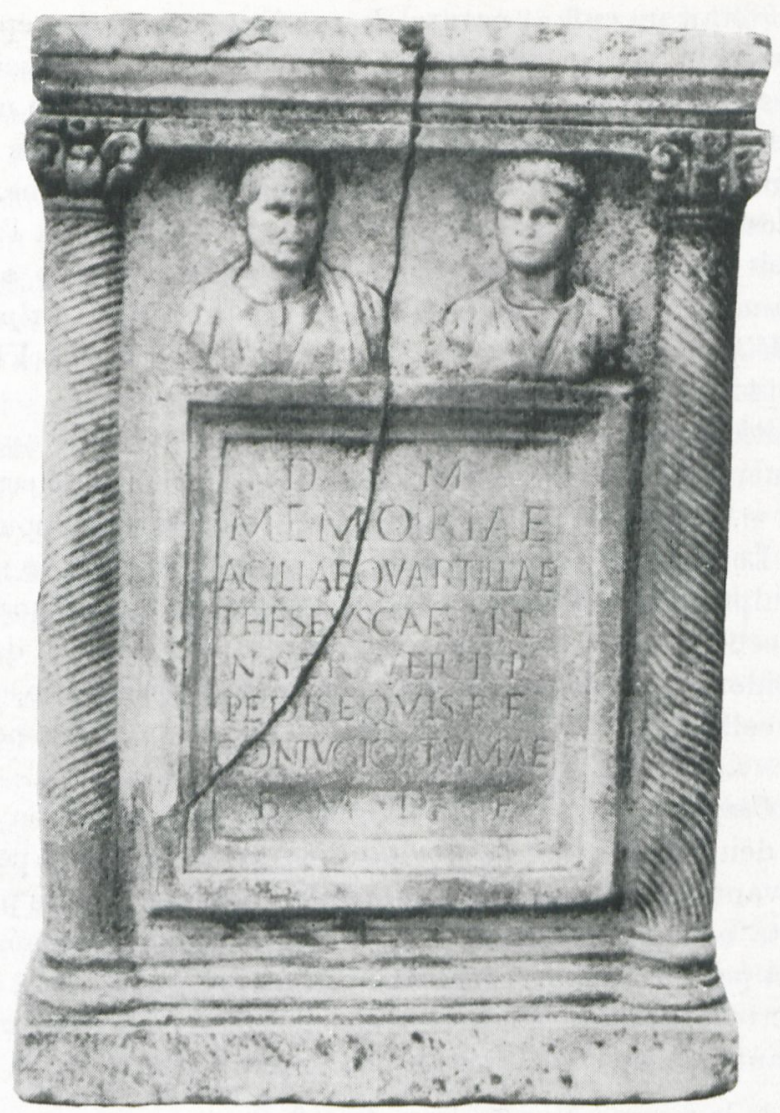

Fig. 2

L'autel servait d'abri pour les cendres, remplissant le rôle de monument et tombeau en même temps. L'inscription se trouve dans un encadrement sur la face frontale, au-dessus d'un soubassement mouluré et au-dessous des portraitsbustes de la défunte et d'e son mari. Dans les angles de l'autel se dressent des colonnettes torses à chapiteaux corinthiens. Les faces latérales sont décorées d'une palmette soutenue par une coquille, exécutés en bas-relief. Similairement aux colonnettes de la face frontale, dans les angles arrière se dressent des faisceaux de trois flambeaux allumés. Une corniche moulurée soutenait le couvercle de l'autel.

Les bustes sont découpés à la hauteur des seins et au-dessous des épaules. Le portrait masculin est à gauche, le féminin à droite. L'homme et la femme sont vêtus de tuniques. Une lacerna aux plis souples couvre l'épaule gauche du mari, une palla celle de sa femme. Les têtes sont légèrement tournées vers le centre du relief, ainsi une oreille reste visible du côté extérieur. Dans le portrait masculin les cheveux courts retombent en mèches sur les tempes tandis que le front est entièrement chauve. La femme est coiffée en couronne très opulente, formée de deux nattes, qui est épinglée tout près du front. 


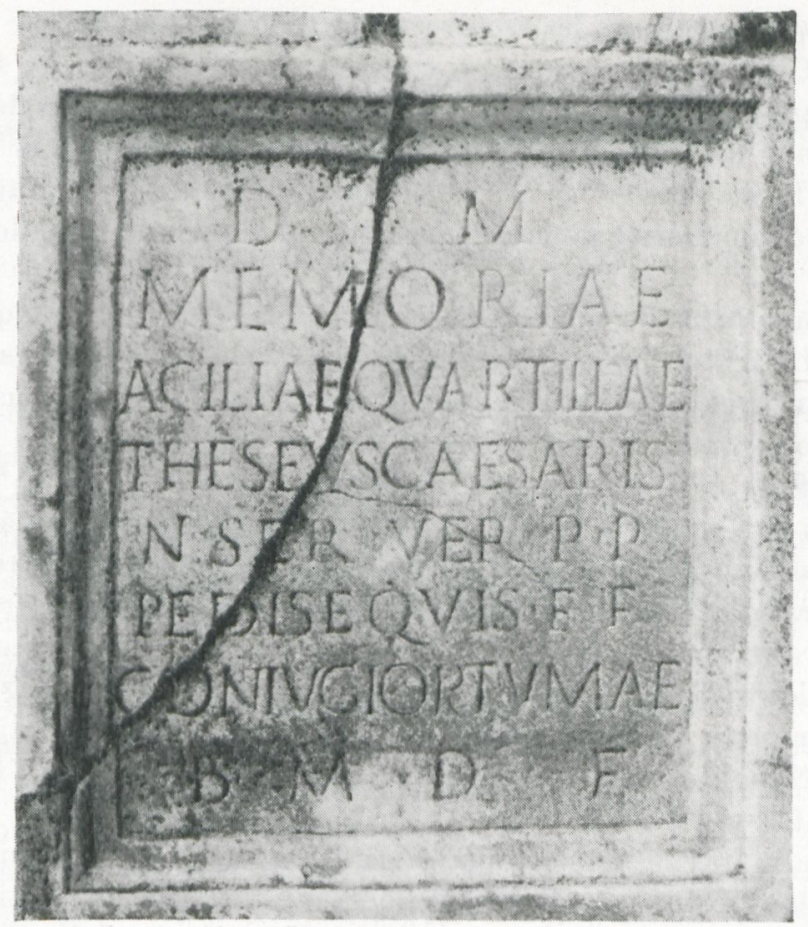

Fig. 3

Texte:

D M

MEMORIAE

ACILIAEQVARTILLAE

THESEVSC ESARIS

$\mathrm{N} \cdot \mathrm{SER} \cdot \mathrm{VER} \cdot \mathrm{P} \cdot \mathrm{P}$

PEDISEQVIS.F.F

CONIVGIOPTVMAE

B M D É

Transeription :

$1 D$ (is) M(anibus).

Memoriae

Aciliae Quartillae

Theseus Caesaris

5 n(ostri) $\operatorname{ser}($ vus ) ver(na) $p$ (rae) $p$ (ositus)

pedisequis $f$ (ieri) $f$ (ecit).

Coniugi opt $\langle i\rangle$ mae

b(ene) m(eritae) d(e) $[s] e ́$ 
Les textes qui concernent les esclaves et jettent une lumière sur leur vie sont plutôt rares. C'est pourquoi nous croyons que le personnage du fondateur de l'autel fait l'épitaphe digne de mention. Il était né en la maison de l'Empereur et il a fait dans cette maison sa carrière, devenant chef d'une catégorie de domestiques. Probablement ce poste a rendu possible son mariage, d'ailleurs illégitime, avec une femme de condition libre, quoique modeste, dont témoigne son portrait.

Il serait important de connaître le nom de l'Empereur auquel Theseus appartenait. Le texte n'éclaircit point ce problème, mais grâce aux portraits du couple nous pouvons ehercher une réponse. La forme des bustes est celle qui caractérise la ronde bosse à l'époque tardive de Trajan et au temps d'Hadrien. ${ }^{1}$ La coiffure de Theseus est caractéristique pour l'époque de Trajan. Celle d'Acilia, assez rare en ronde bosse, est très simple mais ne fut portée par aucune impératrice, ce qui rend la datation difficile. Elle est plus fréquente sur les basreliefs, c'est à dire dans le portrait funéraire, surtout des affranchies. Les portraits en relief ressemblants à celui d'Acilia sont les suivants: sur la stèle funéraire flavienne de M. Postumius Zosimus à la Ny Carlsberg Glyptothek; sur l'autel funéraire de $M$. Antonius Trophimus du temps de Trajan au Museo Nazionale di Napoli et sur la stèle de Sancia Pieris du temps d'Hadrien à la Ny Carlsberg Glyptothek. ${ }^{2}$

La qualité du travail et la composition du relief trouvent leurs meilleures analogies sur les monuments du temps de Trajan. Il faut ici mentionner encore une fois l'autel de M. Antonius Trophimus et la stèle de Julia Saturnina à la Ny Carlsberg Glyptothek, décorée de colonnes torses et de portraits-bustes. ${ }^{3}$

Tous ces détails, ainsi que le modelé linéaire et l'austérité du décor rendent improbable une période précédant la fin du règne de Domitien et dépassant le comencement du règne d'Hadrien. Il reste possible que Theseus appartint à Hadrien ou bien à Trajan, mais probablement il était un verna et praepositus pedisequis de ce dernier.

1 P. Bieńkowski, Note sur l'histoire du buste dans l'Antiquité, RA 27, 1895, 295; G. Daltrop, Die stadtrömischen männlichen Privatbildnisse trajanischer und hadrianischer Zeit, Münster 1958, passim.

2 W. Altmann, Die römischen Grabaltäre der Kaiserzeit, Berlin 1905, resp. nos 271. 282. 277.

${ }^{3}$ Altmann, op. cit. no 276. 